\title{
MORE SMOOTHLY REAL COMPACT SPACES
}

\author{
ANDREAS KRIEGL AND PETER W. MICHOR
}

(Communicated by Andrew M. Bruckner)

\begin{abstract}
A topological space $X$ is called $\mathscr{A}$-real compact if every algebra homomorphism from $\mathscr{A}$ to the reals is an evaluation at some point of $X$, where $\mathscr{A}$ is an algebra of continuous functions. Our main interest lies on algebras of smooth functions. Arias-de-Reyna has shown that any separable Banach space is smoothly real compact. Here we generalize this result to a huge class of locally convex spaces including arbitrary products of separable Fréchet spaces.
\end{abstract}

In [KMS] the notion of real compactness was generalized by defining a topological space $X$ to be $\mathscr{A}$-real-compact if every algebra homomorphism $\alpha: \mathscr{A} \rightarrow$ $\mathbb{R}$ is just the evaluation at some point $a \in X$, where $\mathscr{A}$ is some subalgebra of $C(X, \mathbb{R})$. In case $\mathscr{A}$ equals the algebra $C(X, \mathbb{R})$ of all continuous functions this condition reduces to the usual real-compactness. Our main interest lies on algebras $\mathscr{A}$ of smooth functions. In particular we showed in [KMS] that every space admitting $\mathscr{A}$-partitions of unity is $\mathscr{A}$-real-compact. Furthermore any product of the real line $\mathbb{R}$ is $C^{\infty}$-real-compact. A question we could not solve was whether $\ell^{1}$ is $C^{\infty}$-real-compact, despite the fact that there are no smooth bump functions. [AdR] had already shown that this is true not only for $\ell^{1}$, but for any separable Banach space.

The aim of this paper is to generalize this result of [AdR] to a huge class of locally convex spaces, including arbitrary products of separable Fréchet spaces.

Convention. All subalgebras $\mathscr{A} \subseteq C(X, \mathbb{R})$ are assumed to be real algebras with unit and with the additional property that for any $f \in \mathscr{A}$ with $f(x) \neq 0$ for all $x \in X$ the function $1 / f$ lies also in $\mathscr{A}$.

1. Lemma. Let $\mathscr{A} \subset C(X, \mathbb{R})$ be a finitely generated subalgebra of continuous functions on a topological space $X$. Then $X$ is $\mathscr{A}$-real-compact.

Proof. Let $\alpha: \mathscr{A} \rightarrow \mathbb{R}$ be an algebra homomorphism. We first show that for any finite set $\mathscr{F} \subset \mathscr{A}$ there exists a point $x \in X$ with $f(x)=\alpha(f)$ for all $f \in \mathscr{F}$.

For $f \in \mathscr{A}$ let $Z(f):=\{x \in X: f(x)=\alpha(f)\}$. Then $Z(f)=Z(f-\alpha(f) 1)$, since $\alpha(f-\alpha(f) 1)=0$. Hence we may assume that all $f \in \mathscr{F}$ are even contained in $\operatorname{ker} \alpha=\{f: \alpha(f)=0\}$. Then $\bigcap_{f \in \mathscr{F}} Z(f)=Z\left(\sum_{f \in \mathscr{F}} f^{2}\right)$. The

Received by the editors June 16, 1991.

1991 Mathematics Subject Classification. Primary 46E50, 58A40, 54D60. 
sets $Z(f)$ are not empty, since otherwise $f \in \operatorname{ker} \alpha$ and $f(x) \neq 0$ for all $x$, so $1 / f \in \mathscr{A}$ and hence $1=f / f \in \operatorname{ker} \alpha$, a contradiction to $\alpha(1)=1$.

Now the lemma is valid, whether the condition "finitely generated" is meant in the sense of an ordinary algebra or even as an algebra with the additional assumption on non-vanishing functions, since then any $f \in \mathscr{A}$ can be written as a rational function in the elements of $\mathscr{F}$. Thus $\alpha$ applied to such a rational function is just the rational function in the corresponding elements of $\alpha(\mathscr{F})=$ $\mathscr{F}(x)$ and is thus the value of the rational function at $x$.

2. Corollary. Any algebra-homomorphism $\alpha: \mathscr{A} \rightarrow \mathbb{R}$ is monotone.

Proof. Let $f_{1} \leq f_{2}$. By Lemma 1 there exists an $x \in X$ such that $\alpha\left(f_{i}\right)=f_{i}(x)$ for $i=1,2$. Thus $\alpha\left(f_{1}\right)=f_{1}(x) \leq f_{2}(x)=\alpha\left(f_{2}\right)$.

3. Corollary. Any algebra-homomorphism $\alpha: \mathscr{A} \rightarrow \mathbb{R}$ is bounded, for every convenient algebra structure on $\mathscr{A}$.

By a convenient algebra structure we mean a convenient vector space structure for which the multiplication $\mathscr{A} \times \mathscr{A} \rightarrow \mathscr{A}$ is a bilinear bornological mapping. A convenient vector space is a separated locally convex vector space that is Mackey complete, see [FK].

Proof. Suppose that $f_{n}$ is a bounded sequence, but $\left|\alpha\left(f_{n}\right)\right|$ is unbounded. Replacing $f_{n}$ by $f_{n}^{2}$ we may assume that $f_{n} \geq 0$ and hence also $\alpha\left(f_{n}\right) \geq 0$. Choosing a subsequence we may even assume that $\alpha\left(f_{n}\right) \geq 2^{n}$. Now consider $\sum_{n} f_{n} / 2^{n}$. This series converges in the sense of Mackey, and since the bornology on $\mathscr{A}$ is complete, the limit is an element $f \in \mathscr{A}$. Applying $\alpha$ yields

$$
\begin{aligned}
\alpha(f) & =\alpha\left(\sum_{n=0}^{N} \frac{1}{2^{n}} f_{n}+\sum_{n>N} \frac{1}{2^{n}} f_{n}\right)=\sum_{n=0}^{N} \frac{1}{2^{n}} \alpha\left(f_{n}\right)+\alpha\left(\sum_{n>N} \frac{1}{2^{n}} f_{n}\right) \\
& \geq \sum_{n=0}^{N} \frac{1}{2^{n}} \alpha\left(f_{n}\right)+0=\sum_{n=0}^{N} \frac{1}{2^{n}} \alpha\left(f_{n}\right),
\end{aligned}
$$

where we applied to the function $\sum_{n>N} \frac{1}{2^{n}} f_{n} \geq 0$ that $\alpha$ is monotone. Thus the series $\sum_{n=0}^{N} \alpha\left(f_{n}\right) / 2^{n}$ is bounded and increasing, hence converges, but its summands are bounded by 1 from below. This is a contradiction.

4. Definition. We recall that a mapping $f: E \rightarrow F$ between convenient vector spaces is called smooth $\left(C^{\infty}\right.$ for short) if the composite $f \circ c: \mathbb{R} \rightarrow F$ is smooth for every smooth curve $c: \mathbb{R} \rightarrow E$. It can be shown that under these assumptions derivatives $f^{(p)}: E \rightarrow L^{p}(E, F)$ exist. See [FK].

A mapping is called $C_{c}^{\infty}$ if in addition all derivatives considered as mappings $d^{p} f: E \times E^{p} \rightarrow F$ are continuous.

Now we generalize Lemma 5 and Proposition 7 of [AdR] to arbitrary convenient vector spaces.

5. Definition. Let $\mathscr{A} \subseteq C(X, \mathbb{R})$ be a set of continuous functions on $X$. We say that a space $X$ admits large carriers of class $\mathscr{A}$ if for every neighborhood $U$ of a point $p \in X$ there exists a function $f \in \mathscr{A}$ with $f(p)=0$ and $f(x) \neq 0$ for all $x \notin U$. 
Every $\mathscr{A}$-regular space $X$ admits large $\mathscr{A}$-carriers, where $X$ is called $\mathscr{A}$ regular if for every neighborhood $U$ of a point $p \in X$ there exists a function $f \in \mathscr{A}$ with $f(p)>0$ and $f(x)=0$ for $x \notin U$. The existence of large $\mathscr{A}$-carriers follows by using the modified function $\bar{f}:=f(a)-f$.

In [AdR, Proof of Theorem 8] it is proved that every separable Banach space admits large $C_{c}^{\infty}$-carriers. The carrying functions can even be chosen as polynomials as shown in Lemma 7 below.

6. Lemma. Let $E$ be a convenient vector space, $\left\{x_{n}^{\prime}: n \in \mathbb{N}\right\} \subset E^{\prime}$ be bounded, $\left(\lambda_{n}\right) \in \ell^{1}(\mathbb{N})$. Then the series $(x, y) \mapsto \sum_{n=1}^{\infty} \lambda_{n} x_{n}^{\prime}(x) x_{n}^{\prime}(y)$ converges to $a$ continuous symmetric bilinear function on $E \times E$.

Proof. Clearly the function converges pointwise. Since the sequence $\left\{x_{n}^{\prime}\right\}$ is bounded, it is equicontinuous, hence bounded on some neighborhood $U$ of 0 , so there exists a constant $M \in \mathbb{R}$ such that $\left|x_{n}^{\prime}(U)\right| \leq M$ for all $n \in \mathbb{N}$. For $x, y \in U$ we have $\left|\sum_{n=1}^{\infty} \lambda_{n} x_{n}^{\prime}(x) x_{n}^{\prime}(y)\right| \leq \sum_{n=1}^{\infty}\left|\lambda_{n}\right| M^{2}$, which suffices for continuity of a bilinear function.

7. Lemma. Let $E$ be a Banach space that is separable or whose dual is separable for the topology of pointwise convergence. Then $E$ admits large carriers for continuous polynomials of degree 2.

Proof. If $E$ is separable there exists a dense sequence $\left(x_{n}\right)$ in $E$. By the Hahn-Banach theorem [J, 7.2.4] there exist $x_{n}^{\prime} \in E^{\prime}$ with $x_{n}^{\prime}\left(x_{n}\right)=\left|x_{n}\right|$ and $\left|x_{n}^{\prime}\right| \leq 1$.

Claim. $\sup _{n}\left|x_{n}^{\prime}(x)\right|=|x|$.

Since $\left|x_{n}^{\prime}\right| \leq 1$ we have $(\leq)$. For the converse direction let $\delta>0$ be given. By denseness there exists an $n \in \mathbb{N}$ such that $\left|x_{n}-x\right|<\frac{\delta}{2}$. So we have

$$
\begin{aligned}
|x| & \leq\left|x_{n}\right|+\left|x-x_{n}\right|<\left|x_{n}^{\prime}\left(x_{n}\right)\right|+\frac{\delta}{2} \\
& \leq\left|x_{n}^{\prime}(x)\right|+\underbrace{\left|x_{n}^{\prime}\left(x-x_{n}\right)\right|}_{<\left|x-x_{n}\right|<\frac{\delta}{2}}+\frac{\delta}{2}<\left|x_{n}^{\prime}(x)\right|+\delta .
\end{aligned}
$$

If the dual $E^{\prime}$ is separable for the topology of pointwise convergence, then let $x_{n}^{\prime}$ be a sequence that is weakly dense in the unit ball of $E^{\prime}$. Then $|x|=$ $\sup _{n}\left|x_{n}^{\prime}(x)\right|$.

In both cases the continuous polynomials of Lemma 6

$$
x \mapsto \sum_{n=1}^{\infty} x_{n}^{\prime}(x-a)^{2} / n^{2}
$$

vanish exactly at $a$.

8. Lemma. Let $\alpha: \mathscr{A} \rightarrow \mathbb{R}$ be an algebra homomorphism and assume that some subset $\mathscr{A}_{0} \subset \mathscr{A}$ exists and a point $a \in X$ such that $\alpha\left(f_{0}\right)=f_{0}(a)$ for all $f_{0} \in \mathscr{A}_{0}$ and such that $X$ admits large carriers of class $\mathscr{A}_{0}$.

Then $\alpha(f)=f(a)$ for all $f \in \mathscr{A}$.

Proof. Let $f \in \mathscr{A}$ be arbitrary. Since $X$ admits large $\mathscr{A}_{0}$-carriers, there exists for every neighborhood $U$ of $a$ a function $f_{U} \in \mathscr{A}_{0}$ with $f_{U}(a)=0$ and $f_{U}(x) \neq 0$ for all $x \in U$. By Lemma 1 there exists a point $a_{U}$ such that $\alpha(f)=f\left(a_{U}\right)$ and $\alpha\left(f_{U}\right)=f_{U}\left(a_{U}\right)$. Since $f_{U} \in \mathscr{A}_{0}$, we have $f_{U}\left(a_{U}\right)=$ 
$\alpha\left(f_{U}\right)=f_{U}(a)=0$, hence $a_{U} \in U$. Thus the net $a_{U}$ converges to $a$ and consequently $f(a)=f\left(\lim _{U} a_{U}\right)=\lim _{U} f\left(a_{U}\right)=\lim _{U} \alpha(f)=\alpha(f)$ since $f$ is continuous.

Now we generalize Proposition 2 and Lemma 3 of [BBL]. For every convenient vector space $E$, let a subalgebra $\mathscr{A}(E)$ of $C(E, \mathbb{R})$ be given, such that for every $f \in L(E, F)$ the image of $f^{*}$ on $\mathscr{A}(F)$ lies in $\mathscr{A}(E)$. Examples are $C_{c}^{\infty}, C^{\infty} \cap C, C_{c}^{\omega}:=C_{c}^{\infty} \cap C^{\omega}, C^{\omega} \cap C$, where $C^{\omega}$ denotes the algebra of real analytic functions in the sense of $[\mathrm{KM}]$ and suitable algebras of functions of finite differentiability like $\mathrm{Lip}^{m}$ (see [FK]) or $C_{c}^{m}$.

9. Theorem. Let $E_{i}$ be $\mathscr{A}$-real-compact spaces that admit large carriers of class $\mathscr{A}$. Then any closed subspace of the product of the spaces $E_{i}$ and, in particular, every projective limit of these spaces, has the same properties.

Proof. First we show that this is true for the product $E$. We use Lemma 8 with $\mathscr{A}(E)$ for $\mathscr{A}$ and the vector space generated by $\bigcup_{i}\left\{f \circ \operatorname{pr}_{i}: f \in \mathscr{A}\left(E_{i}\right)\right\}$ for $\mathscr{A}_{0}$, where $\operatorname{pr}_{j}: E=\prod_{i} E_{i} \rightarrow E_{j}$ denotes the canonical projection. Let the finite sum $f=\sum_{i} f_{i} \circ \mathrm{pr}_{i}$ be an element of $\mathscr{A}_{0}$. Since $\alpha \circ \mathrm{pr}_{i}{ }^{*}: \mathscr{A}\left(E_{i}\right) \rightarrow$ $\mathscr{A}(E) \rightarrow \mathbb{R}$ is an algebra homomorphism, there exists a point $a_{i} \in E_{i}$ such that $\alpha\left(f_{i} \circ \mathrm{pr}_{i}\right)=\left(\alpha \circ \operatorname{pr}_{i}{ }^{*}\right)\left(f_{i}\right)=f_{i}\left(a_{i}\right)$. Let $a$ be the point in $E$ with coordinates $a_{i}$. Then

$$
\alpha(f)=\alpha\left(\sum_{i} f_{i} \circ \mathrm{pr}_{i}\right)=\sum_{i} \alpha\left(f_{i} \circ \mathrm{pr}_{i}\right)=\sum_{i} f_{i}\left(a_{i}\right)=\sum_{i}\left(f_{i} \circ \mathrm{pr}_{i}\right)(a)=f(a) .
$$

Now let $U$ be a neighborhood of $a$ in $E$. Since we consider the product topology on $E$, we may assume that $a \in \Pi U_{i} \subset U$, where $U_{i}$ are neighborhoods of $a_{i}$ in $E_{i}$ and are equal to $E_{i}$ except for $i$ in some finite subset $F$ of the index set. Now choose $f_{i} \in \mathscr{A}\left(E_{i}\right)$ with $f_{i}\left(a_{i}\right)=0$ and $f_{i}\left(x_{i}\right) \neq 0$ for all $x_{i} \notin U_{i}$. Consider $f=\sum_{i \in F}\left(f_{i} \circ \mathrm{pr}_{i}\right)^{2} \in \mathscr{A}_{0}$. Then $f(a)=\sum_{i \in F} f_{i}\left(a_{i}\right)^{2}=0$. Furthermore $x \notin U$ implies that $x_{i} \notin U_{i}$ for some $i$, which turns out to be in $F$, and hence $f(x) \geq f_{i}\left(x_{i}\right)^{2}>0$. So we may apply Lemma 8 to conclude that $\alpha(f)=f(a)$ for all $f \in \mathscr{A}(E)$.

Now we prove the result for a closed subspace $F \subset E$. Again we want to apply Lemma 8 , this time with $\mathscr{A}(F)$ for $\mathscr{A}$ and $\left\{\left.f\right|_{F}: f \in \mathscr{A}(E)\right\}$ for $\mathscr{A}_{0}$. Since $\alpha \circ$ incl $^{*}: \mathscr{A}(E) \rightarrow \mathscr{A}(F) \rightarrow \mathbb{R}$ is an algebra homomorphism there exists an $a \in E$ with $\alpha\left(\left.f\right|_{F}\right)=f(a)$ for all $f \in \mathscr{A}(E)$. Now let $U$ be a neighborhood of $a$ in $E$; then there exists an $f_{U} \in \mathscr{A}(E)$ with $f_{U}(a)=0$ and $f_{U}(x) \neq 0$ for all $x \notin U$. By Lemma 1 there exists a point $a_{U} \in F$ such that $f_{U}\left(a_{U}\right)=\alpha\left(\left.f_{U}\right|_{F}\right)=f_{U}(a)=0$. Hence $a_{U}$ is in $U$, and thus is a net in $F$ that converges to $a$. In particular, $a \in F$ since $F$ is closed in $E$. If $V$ is a neighborhood of $a$ in $F$ then there exists a neighborhood $U$ of $a$ in $E$ with $U \cap F \subset V$ and hence an $f \in \mathscr{A}_{0}$ with $f(a)=0$ and $f(x) \neq 0$ for all $x \notin U$. So again Lemma 8 applies.

10. Remark. Theorem 9 shows that a closed subspace of a product of certain $\mathscr{A}$-real-compact spaces is again $\mathscr{A}$-real-compact. Of course the natural question arises of whether the result remains true for arbitrary $\mathscr{A}$-real-compact spaces.

The question is even open-whether the product of two $\mathscr{A}$-real-compact spaces is $\mathscr{A}$-real-compact, or whether a closed subspace of an $\mathscr{A}$-real-compact 
space is $\mathscr{A}$-real-compact, or whether a projective limit of a projective system of $\mathscr{A}$-real-compact spaces is $\mathscr{A}$-real-compact.

11. Corollary. Let $E$ be a separable Fréchet space (e.g., a Fréchet-Montel space); then every algebra homomorphism on $C^{\infty}(E, \mathbb{R})$ or on $C_{c}^{\infty}(E, \mathbb{R})$ is a point evaluation. The same is true for any product of separable Fréchet spaces.

Proof. Any Fréchet space has a countable Basis $\mathscr{U}$ of absolutely convex 0neighborhoods, and since it is complete, it is a closed subspace of the product $\prod_{u \in \mathscr{U}} \widetilde{E_{(U)}}$. The $E_{(U)}$ are the normed spaces formed by $E$ modulo the kernel of the Minkowski functional generated by $U$. As quotients of $E$ the spaces $E_{(U)}$ are separable if $E$ is such. So the completion $\widetilde{E_{(U)}}$ is a separable Banach space, and hence by [AdR, Theorem 8] $\widetilde{E_{(U)}}$ is $C_{c}^{\infty}$-real-compact and admits large $C_{c}^{\infty}$-carriers. By Theorem 9 the same is true for the given Fréchet space. So the result is true for $C_{c}^{\infty}(E, \mathbb{R})$. Since $E$ is metrizable this algebra coincides with $C^{\infty}(E, \mathbb{R})$, see $[\mathrm{K}, 82]$.

Now for a product $E$ of metrizable spaces the two algebras $C^{\infty}(E, \mathbb{R})$ and $C_{c}^{\infty}(E, \mathbb{R})$ again coincide. This can be seen as follows. For every countable subset $A$ of the index set, the corresponding product is separable and metrizable, hence $C^{\infty}$-real-compact. Thus there exists a point $x_{A}$ in this countable product such that $\alpha(f)=f\left(x_{A}\right)$ for all $f$ that factor over the projection to that countable subproduct. Since for $A_{1} \subset A_{2}$ the projection of $x_{A_{2}}$ to the product over $A_{1}$ is just $x_{A_{1}}$ (use the coordinate projections composed with functions on the factors for $f$ ), there is a point $x$ in the product whose projection to the subproduct with index set $A$ is just $x_{A}$. Every Mackey continuous function and, in particular, every $C^{\infty}$-function, depends only on countably many coordinates, thus factors over the projection to some subproduct with countable index set $A$, hence $\alpha(f)=f\left(x_{A}\right)=f(x)$. This can be shown by the same proof as for a product of factors $\mathbb{R}$ in [FK, Theorem 6.2.9] since the result of [M, 1952] is valid for a product of separable metrizable spaces.

\section{REFERENCES}

[BBL] Peter Biström, Sten Bjon, and Mikael Lindström, Homomorphisms on some function algebras, Monatsh. Math. 11 (1991), 93-97.

[FK] Alfred Frölicher and Andreas Kriegl, Linear spaces and differentiation theory, Pure Appl. Math., Wiley, Chichester, 1988.

[K] Andreas Kriegl, Eine kartesisch abgeschlossene Kategorie glatter Abbildungen zwischen bel iebigen lokalkonvexen Vektorräumen, Monatsh. Math. 95 (1983), 287-309.

[KM] Andreas Kriegl and Peter W. Michor, A convenient setting for real analytic mappings, Acta Math. 165 (1990), 105-159.

[KMS] Andreas Kriegl, Peter W. Michor, and Walter Schachermayer, Characters on algebras of smooth functions, Ann. Global Anal. Geom. 7 (1989), 85-92.

[M] S. Mazur, On continuous mappings on cartesian products, Fund. Math. 39 (1952), 229-238.

[AdR] Juan Arias-de-Reyna, A real valued homomorphism on algebras of differentiable functions, Proc. Amer. Math. Soc. 104 (1988), 1054-1058.

Institut für Mathematik, Universität Wien, Strudlhofgasse 4, A-1090 Wien, Austria E-mail address: KRIEGL@AWIRAP.BITNET

E-mail address: MICHOR@AWIRAP.BITNET 